C5s

Journal of Orgonometallic Chemistry, 113 (1976) C58-C60

Elsevier Sequoia S.A., Lausanne - Printed in The Netherlands

\title{
Preliminary communication
}

\section{OPTISCH AKTIVE ÜBERGANGSMETALLKOMPLEXE}

\section{XXXIX*. STEFEOSPEZIFISCHE PHENYL-ADDITION AN DAS $\left.\mathrm{C}_{5} \mathrm{H}_{5}: \mathrm{CO}\right)_{2}$ MO-PYRIDIS-CARBALDININ-KATION}

HENRI BRUNNER* und JOACHIM WACHTER

Institut fur Chemie der Lniversitat Regensburg (Deutschiand)

(Eingegangen den 26. April 1976)

\section{Summary}

The addition of $\mathrm{C}_{6} \mathrm{H}_{5}{ }^{-}$to the $\mathrm{NN}^{\prime}$ ligand $\left(\mathrm{NN}^{\prime}=\right.$ Schiff base of pyridine carbaldehyde- $(2)$ and $(S)-(-)-\alpha-$ phenylethylamine $)$ in $\left[C_{5} H_{5} M O(C O)_{2} N^{*}\right] P F_{6}$ occurs stereospecific from the side opposite to the $\mathrm{C}_{5} \mathrm{H}_{5}$-ring.

Die kationischen Pyridin-carbaldimin-Komplexe $I$ addieren Nukleophile, $\mathrm{X}=\mathrm{H}^{-}, \mathrm{C}_{6} \mathrm{H}_{5}{ }^{-}$usw., am Azomethinkohlenstoffatom der $\mathrm{C}-\mathrm{N}$-Doppelbindung [2]. Dabei bildet sich fur $\mathrm{X} \neq \mathrm{H}^{-}$ein neues Chiralitatszentrum im Chelatliganden. Mit Hilfe des optisch reinen Komplexes $(-)_{365}-\left[\mathrm{C}_{5} \mathrm{H}_{5}\right.$ Mo$\left.(\mathrm{CO})_{2} \mathrm{NN}^{\prime}\right] \mathrm{PF}_{6}$ I $\left(\mathrm{NN}^{\prime}=\right.$ Schiffloase aus Pyridin-carbaldehyd-2 unc $S-(-)-\alpha-$ Phenyläthylamin) bekannter absoluter Konfiguration $[3,4]$ sollte gepruft werden, ob die Phenyladdition am Azomethinkohlenstoffatom bevorzugt von der dem $\mathrm{C}_{s} \mathrm{H}_{s}$-Rest zugewandten oder abgewandten Seite erfolgt und wie hoch das Ausmass der Stereoselektivitat bei dieser Reaktion ist.

Bei der Umsetzung des optiseh reinen Komplexes $1,[\alpha]_{365}^{20}-4150^{\circ}$, mit Phenyllithium erhält man in $22 \%$ iger Ausbeute den orangeroten Neutralkomplex II, $[\alpha]_{365}^{20}+4525^{\circ}$. II zeigt im IR-Spektrum zwei Carbonylbanden bei 1920 und $1815 \mathrm{~cm}^{-1}$.

Im Massenspektrum tritt bei $m / e 506$ der Molekülpeak der ${ }^{98}$ Mo-Spezies auf. Das ${ }^{1} \mathrm{H}$-NMR-Spektrum enthält für die $\mathrm{C}_{5} \mathrm{H}_{5}$-Protonen nur ein scharfes Singulett bei $\tau$ 4.93 ppm und fur die Methylgruppe nur ein Dublett bei - $8.29 \mathrm{ppm}\left(\mathrm{J}\left(\mathrm{CH}_{3}-\mathrm{CH}\right) \hat{0} .5 \mathrm{~Hz}\right)$.

In den bisher untersuchten Diastereomerenpaaren $\mathrm{C}_{5} \mathrm{H}_{5} \mathrm{Mo}(\mathrm{CO})_{2} \mathrm{LL}^{\prime}$ mit entgegengesetzter Konfiguration am Metallatom unterscheiden sich die Diastereomeren in ihren ${ }^{1} \mathrm{H}-\mathrm{NMR}$-Spektren $[5,6]$. Daher schliessen wir aus dem 'H-NMR-Spektrum von II, dass bei der Phenyladdition an I stereospezifisch

mxxVII Mitreil siehe Ret. 1. 

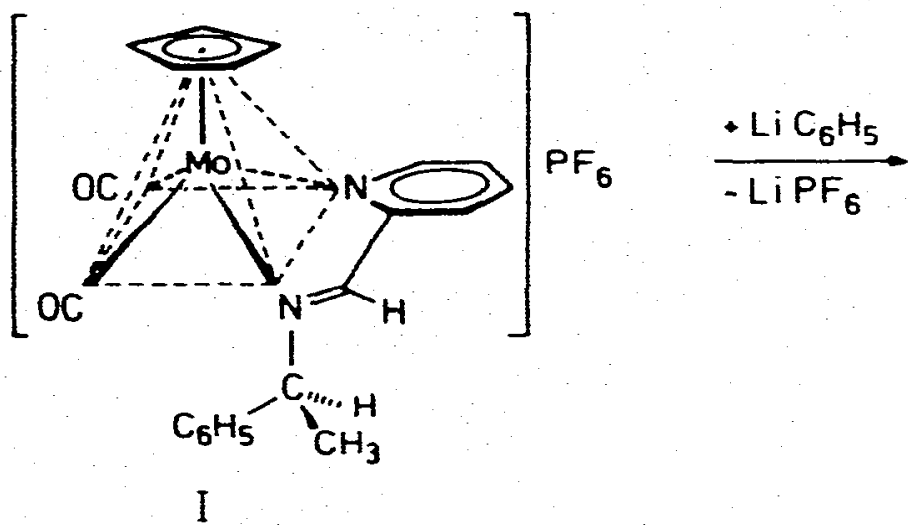

$$
|a|_{365}^{20}-4150^{\circ}
$$

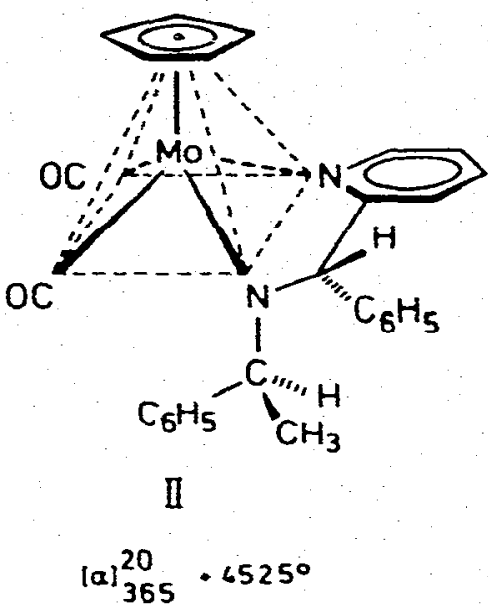

nur eines der beiden moglichen Derivate gebildet wird. Aufgrund von Modellvorstellungen ordnen wir ihm Struktur $11 \mathrm{zu}$, in der die Phenyladdition ausschliesslich von der dem $\mathrm{C}_{s} \mathrm{H}_{5}$-Ring abgewandten Seite erfolgt ist.

Samtliche bisher dargestellten optisch aktiven Komplexe des Typs $\mathrm{C}_{5} \mathrm{H}_{5} \mathrm{Mo}(\mathrm{CO})_{2} \mathrm{LL}^{\prime}$ epimerisieren in Lösung durch Konfigurationsanderung am Mo-Atom [5-7]. Die Geschwindigkeit dieser intramolekularen Umlagerung.

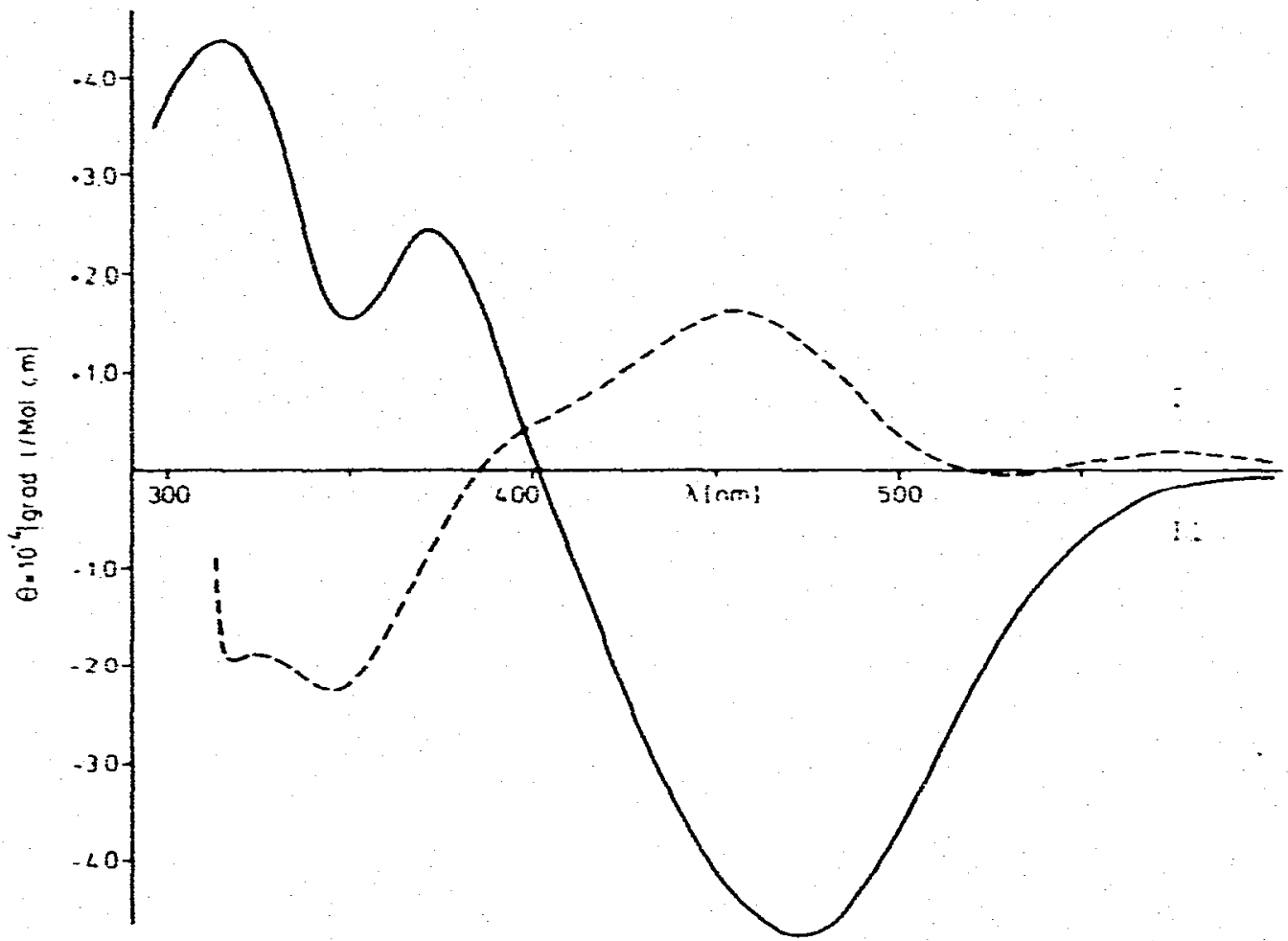

Fig. 1. CD-Spektren von $1\left(10^{-3} M\right.$ in Aceton) und $11\left(10^{-3} M\right.$ in Toluol). 
die polarimetrisch oder ${ }^{1} \mathrm{H}$-NMIR-spektroskopisch verfolgt werden kann, ist stark von den Chelatliganden $L^{\prime} L^{\prime}$ abhangig $[5,6]$. Die Epimerisierung führt zu Gleichgewichten. in denen eines der beiden Diastereomeren stark dominieren kann [8]. Dieses Verhalten ist für II nicht zu beobachten. Auch beim Erhitzen von II in Toluol-Losung auf $90^{\circ} \mathrm{C}$ ist weder eine Abnahme der Drehwerte noch ein Auftreten neuer ${ }^{1} \mathrm{H}-\mathrm{NM}$ R-Signale für das Isomere mit der zu II spiegelbildlichen Konfiguration am Mo-Atom zu erkennen.

Auffallend ist. dass I und II entgegengesetzf.e Drehwerte und CD-Spektren hiben (Fig. 1). Da bei der Reaktion von I mit $\mathrm{LiC}_{6} \mathrm{H}_{5}$ die Konfiguration am Mo-Atom erhalten bleibt, ist dies auf cie Umwandlung des Imin-Stickstoffs in $I$ in den Amido-Stickstoff in II zurickzufuhren.

\section{Arbeitsvorschrift}

Die Suspension von $1.1 \mathrm{~g}(1.9 \mathrm{mmol}) \mathrm{I}$ in $100 \mathrm{ml}$ Äther wird bei $-78^{\circ} \mathrm{C} \mathrm{mit}$ $1.9 \mathrm{mmol} \mathrm{C}_{6} \mathrm{H}_{5} \mathrm{Li}$, gelost in $10 \mathrm{ml} \ddot{A}$ ther, versetzt und nach langsamem Erwärmen auf Raumtemperatur 4 Std. geruhrt. Das Komplexsalz I geht dabei in Losung. Die Chromatographie an $\mathrm{SiO}_{2}$ mit Benzol liefert $210 \mathrm{mg}(22 \%)$ II in Form eines orangen Pulvers. Schmp. $149^{\circ} \mathrm{C}$ (Zers.). (Gef.: C, 64.17; H, 4.80; N, 5.55; Mol.-Gew. (massenspektrometrisch) 506 (bzgl. ${ }^{98} \mathrm{Mo}$ ). $\mathrm{C}_{2} ; \mathrm{H}_{24} \mathrm{~N}_{2} \mathrm{O}_{2}$ Mo ber.: C, 64.29; H, 4.79; N, 5.55\%; Mol.-Gew. 504.4).

\section{Dank}

Diese Arbeit wurde von der Deutschen Forschungsgemeinschaft und dem Fonds der Chemischen Industrie unterstutzt. Der Firma Dynamit Nobel AG danken wir fur Überlassung von $S-(-)-\alpha$-Phenylathylamin.

\section{Literatur}

1 H. Brunner und 3. Wachter, Angew: Chem.. im Druck.

2 J. Wachter. Dissertazion. Universität Regensburz. 1976.

3 H. Brunner und W.A. Hermann. Chem. Ber., 105 (1972) 3600.

4 S.J. LuPlaca. L. Bermal H. Brunner und $\mathbf{W}$.A. Hezrann, Angew. Chem.. 87 (1975) 379:

Angew. Chem Int. Ed. End. 14 (1975) 353.

5 H. Brunzer. Top. Curr. Chem. 56 (1975) 67.

6 H. Brunner und J. Wachter. J. Orzanometal Chem.. 107 (1976) 307.

7. F. Pfeiffer. J. Kuy per und K. Vrieze. J. Orsanometal. Chem., 105 (1976) 371.

8 H. Brinner. W.A. Hermenn und J. Wachter. J. Organomeral. Chem., 107 (1976) C11. 